\title{
Substorm time scales from polar cap convection measurements
}

\author{
P. T. Jayachandran ${ }^{1}$ and J. W. MacDougall ${ }^{2}$ \\ ${ }^{1}$ Physics Department, University of New Brunswick, Fredericton, New Brunswick, Canada \\ ${ }^{2}$ Department of Physics and Astronomy, University of Western Ontario, London, Ontario, Canada \\ (Received December 20, 2006; Revised June 13, 2007; Accepted June 18, 2007; Online published August 8, 2007)
}

\begin{abstract}
A recent study by Jayachandran et al. (Geophys. Res. Lett., 30, 2064, 2003) reported repeated and detectable changes in the polar cap convection associated with substorms. We report here our use of ionospheric convection measurements to determine the time scales of growth, expansion, and recovery/reconfiguration associated with the substorms. The average time scales, determined from polar cap convection, associated with growth, expansion, and recovery are 31.6, 22.4, and $38.8 \mathrm{~min}$, respectively. A comparison of the growth and expansion time scales determined from the convection measurements with those determined from the geosynchronous satellite measurements revealed interesting differences. The growth time scale determined from the ionospheric convection measurement is smaller than that of the time scale determined from geosynchronous measurements, whereas the expansion time scale determined using the ionospheric convection is much larger than that of the time scale determined using the geosynchronous measurements. The implications of the results in the MagnetosphereIonosphere (M-I) coupling are discussed.
\end{abstract}

Key words: Magnetosphere-ionosphere interactions, plasma convection, polar cap phenomena, storms and substorms, polar ionosphere.

\section{Introduction}

Auroral substorms are a manifestation of the Solar Wind-Magnetosphere-Ionosphere (SW-M-I) coupling during which an enormous amount of energy derived from the solar wind-magnetosphere interaction is episodically deposited in the terrestrial upper atmosphere. Based on ground-based optical and magnetometer observations, it is known that the substorms show three distinct phases, namely, growth, expansion, and recovery. The average time scales associated with these three phases are approximately $1 \mathrm{~h}$, tens of minutes, and $30 \mathrm{~min}$ up to a total of about $3 \mathrm{~h}$ (the individual events show a high degree of variability) (Akasofu, 1964; Kawano et al., 2002). There have been only a few quantitative studies of the time scales associated with substorms (Weimer, 1994; Chua et al., 2004). Chua et al. (2004) used the hemispheric power derived from POLAR UVI to determine the time scales associated with the expansion and recovery phases of the substorm, whereas Weimer (1994) used the ground-based magnetometrically derived indices ( $\mathrm{AU}, \mathrm{AL}$, and $\mathrm{AE}$ ) to determine the time scales associated with the expansion and recovery phases of substorms. None of the above-mentioned studies compared the times scales derived with the inner magnetospheric measurements, such as the geosynchronous satellite measurements.

A recent study by Jayachandran et al. (2003) revealed that polar cap convection shows systematic changes associated with the three phases of substorms. Their study used

Copy right(c) The Society of Geomagnetism and Earth, Planetary and Space Sciences (SGEPSS); The Seismological Society of Japan; The Volcanological Society of Japan; The Geodetic Society of Japan; The Japanese Society for Planetary Sciences; TERRAPUB. steady southward interplanetary magnetic field (IMF) intervals in order to eliminate the IMF variations that would affect the convection. In this study we use the same database and some additional data to determine the time scales of substorms and compare these time scales with the time scales determined from geosynchronous magnetic field measurements.

\section{Data and Method of Analysis}

We have used the convection measured by a Canadian Advanced Digital Ionosonde (CADI) at Resolute Bay (CGM Lat. $83.3^{\circ} \mathrm{N}$, Long. $319^{\circ} \mathrm{E}$ ) situated well within the polar cap. CADI measures the convection velocity (speed and azimuth) every $30 \mathrm{~s}$. Since the primary driver of the convection in the polar cap is the north-south $(\mathrm{Bz})$ component of the IMF (e.g. MacDougall and Jayachandran, 2001), we have selected days when the IMF component is dominantly southward and relatively steady. This criterion will eliminate IMF-induced variations in the polar cap convection. We have also used, whenever available, complementary measurements from POLAR UVI, geosynchronous satellites (GOES 8 and 10), LANL particle measurements, and ground-based magnetometers to determine the substorm onset and subsequent evolution. The basic substorm signature we show in this paper is the dipolarization signature seen in the geosynchronous magnetic field measurements (indicated by a sudden change in the geosynchronous magnetic field inclination).

A typical example representing the variation of the geosynchronous magnetic field inclination determined using the magnetic field measurements from the GOES 10 satellite and the convection measured at Resolute Bay for a substorm on 30 September 2000 is shown in Fig. 1. Fig- 

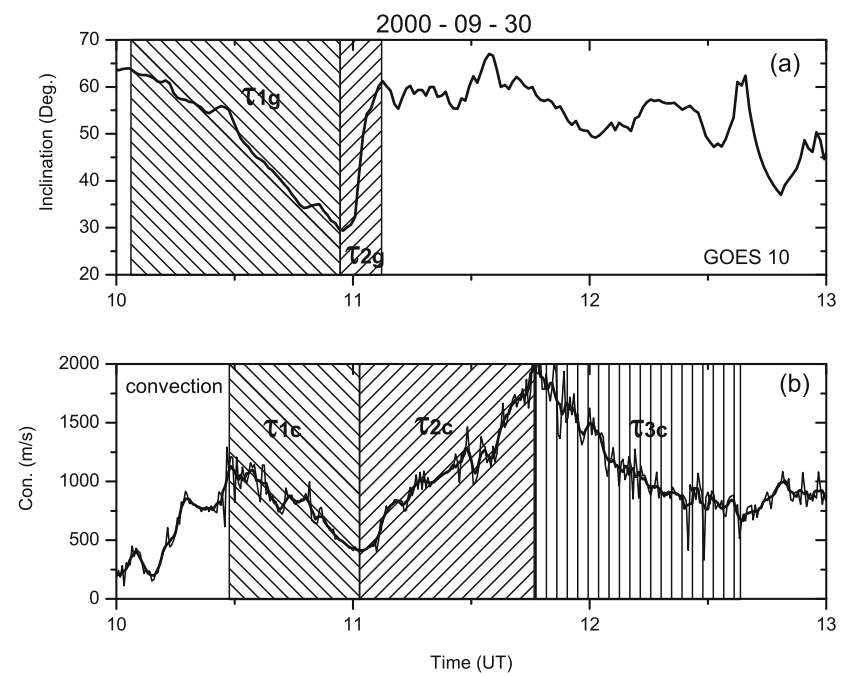

Fig. 1. Variation of (a) magnetic field inclination calculated using the magnetic field measurements from GOES 10 geosynchronous satellite, and (b) ionospheric convection measured at Eureka for 30 September 2000. Shaded regions in the figure represent the time intervals used to determine the time period using an exponential fit.

ure 1(a) represents the geosynchronous magnetic field inclination for the period 10-13 UT, and Fig. 1(b) represents the variation in polar cap convection for the same period. For this period, IMF Bz was southward with the value of approximately $-11 \mathrm{nT}$, and the IMF and solar wind were steady. The substorm onset occurred around approximately 1055 UT and is clearly seen as a rapid increase in the geosynchronous magnetic field inclination (dipolarization) as the field becomes more dipole-like. One can also clearly see the initial decrease in the inclination during the growth phase up to the onset due to the stretching of the tail. Jayachandran et al. (2003) reported a three-stage response of the polar cap convection associated with the substorm as an initial decrease in the convection until the end of the growth phase, a sudden decrease in convection at or after the onset, and a rapid increase in convection shortly after the onset. This three-stage response is clearly seen in the convection time series shown in Fig. 1(b). The convection started decreasing gradually from 1028 UT to approximately 10:50 UT. From 1050 UT to approximately 1100 UT, convection showed a sudden decrease and the convection increased from 1100 UT to approximately 1148 UT after the onset and reached its peak value approximately 1148 UT. An additional stage, which we will consider for this study, is the gradual decrease of the convection from approximately 1148 UT (maximum in convection after the onset) to approximately 1240 UT (approximately pre-substorm value of convection). Since the sudden decrease in convection occurs only for few minutes just before the onset (Jayachandran et al., 2003), we have combined stages 1 and 2 reported by Jayachandran et al. (2003) for this study. As a visual aid, we have marked two time periods in the GOES 10 inclination and three periods in the convection. The time periods $\tau_{1 \mathrm{~g}}$, and $\tau_{2 \mathrm{~g}}$ shown in Fig. 1(a) are the growth and expansion phase time periods from the GOES satellite measurements, respectively. Similarly, time periods $\tau_{1 \mathrm{c}}, \tau_{2 \mathrm{c}}$, and $\tau_{3 \mathrm{c}}$ shown in Fig. 1(b) are growth, expansion, and recovery phase time
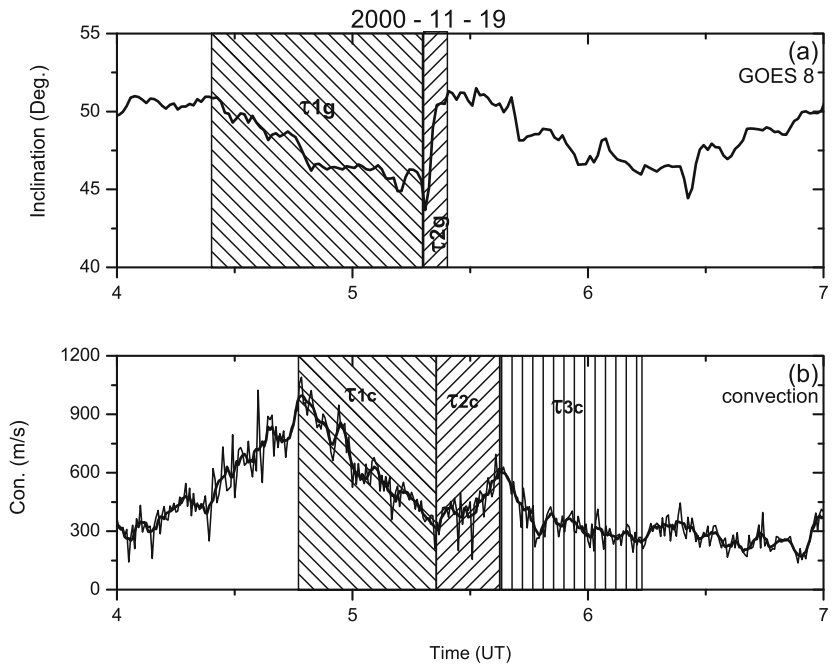

Fig. 2. Variation of (a) magnetic field inclination calculated using the magnetic field measurements from GOES 8 geosynchronous satellite, and (b) ionospheric convection measured at Eureka for 19 November 2000. Shaded regions in the figure represent the time intervals used to determine the time period using an exponential fit.

periods determined from the convection measurements, respectively. We have determined the time period by taking the time difference between the different phases and obviously certain amount of subjectivity is inherent in this method. We have also tried to fit an exponential to determine the time constant of the recovery phase and obtained a similar time constant. We could not determine the recovery time period for the GOES satellite measurements because the field inclination did not show any obvious recovery phase. It is interesting to note that the growth time of the ionospheric convection is shorter than the growth time of the geosynchronous magnetic field, and the expansion time of the ionospheric convection is much longer than the expansion time of the geosynchronous magnetic field.

Another example of the convection and geosynchronous magnetic field inclination changes associated with a substorm is shown in Fig. 2. The format used for this figure is the same as in Fig. 1 except that the onset occurred around 0520 UT of 19 November 2000, and we have used GOES 8 instead of the GOES 10 measurements. IMF $\mathrm{Bz}$ was southward and remained constant at about approximately $-4 \mathrm{nT}$ during the entire duration of the event and the solar wind also remained steady. The variations in convection and GOES 8 inclination are similar to the one described above. Here also the growth time of the ionospheric convection is shorter than the growth time of the geosynchronous magnetic field, and the expansion time of the ionospheric convection is much longer than the expansion time of the geosynchronous magnetic field.

\section{Results and Discussion}

It is obvious from the above two examples that there is a clear and detectable pattern in the polar cap convection and the geosynchronous magnetic field inclination associated with substorms. We have studied 26 substorm events in detail. These substorms occurred during steady southward IMF $\mathrm{Bz}$ and solar wind conditions. For these 26 events, 

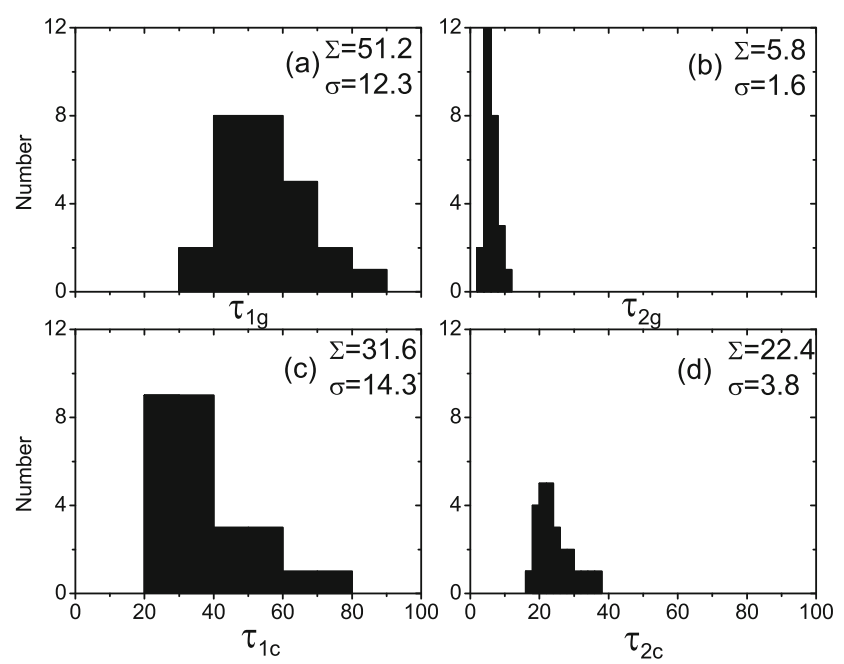

Fig. 3. Distribution of time constants (a) $\tau_{1 \mathrm{~g}}$ (growth-GOES), (b) $\tau_{2 \mathrm{~g}}$ (expansion-GOES), (c) $\tau_{1 \mathrm{c}}$ (growth-convection), and (d) $\tau_{2 \mathrm{c}}$ (expansion-convection). The mean and standard deviation of each distribution are shown in respective figures.

we have calculated the time constants $\tau_{1 \mathrm{~g}}$ (growth) and $\tau_{2 \mathrm{~g}}$ (expansion) from the geosynchronous magnetic field measurements and $\tau_{1 \mathrm{c}}$ (growth), $\tau_{2 \mathrm{c}}$ (expansion), and $\tau_{3 \mathrm{c}}$ (recovery or reconfiguration) from the convection by taking the raw time differences between the time segments shown in Figs. 1 and 2.

Distribution of the time scales $\tau_{1 \mathrm{~g}}, \tau_{2 \mathrm{~g}}, \tau_{1 \mathrm{c}}$, and $\tau_{2 \mathrm{c}}$ are shown in Figs. 3(a)-(d), respectively. The mean and standard of each distribution are shown in the figures. The growth time determined from the GEOS satellite $\left(\tau_{1 \mathrm{~g}}\right)$ is larger $(\Sigma=51.2 \mathrm{~min})$ than the growth time $\left(\tau_{1 \mathrm{c}}\right)$ determined from the convection $(\Sigma=31.5 \mathrm{~min})$. This is also evident in the examples shown in Figs. 1 and 2 where the duration of the reduction in convection associated with the growth phase is much smaller than the duration of reduction in the geosynchronous field inclination. This implies that there is a delay in the growth phase signature seen in the convection. The distribution of the expansion time scales determined from the GOES and convection shows the opposite trend. The expansion time $\left(\tau_{2 \mathrm{~g}}\right)$ determined from the GOES satellite is smaller ( $\Sigma=5.8 \mathrm{~min})$ than the expansion time $\left(\tau_{2 \mathrm{c}}\right)$ determined from the convection measurements ( $\Sigma=22.4 \mathrm{~min}$ ). This implies that the ionospheric response time is much larger than the inner magnetospheric response time. This expansion time distribution determined using the ionospheric convection $\left(\tau_{2 \mathrm{c}}\right)$ is similar to the distribution shown by Chua et al. (2004) using the Polar UVI hemispheric power variation associated with substorms. The difference between time scales determined using the polar cap convection and the geosynchronous magnetic field measurements for growth and expansion phases is almost the same (approx. $19 \mathrm{~min}$ for growth and approx. $17 \mathrm{~min}$ for expansion) and implies that this time (approx. $18 \mathrm{~min}$ ) is inherent to the M-I coupling.

The other time scale, $\tau_{3 \mathrm{c}}$, is the recovery or reconfiguration time and represents the return of the convection to its pre-substorm value. This can only be determined from the convection since most of the GOES inclination does not

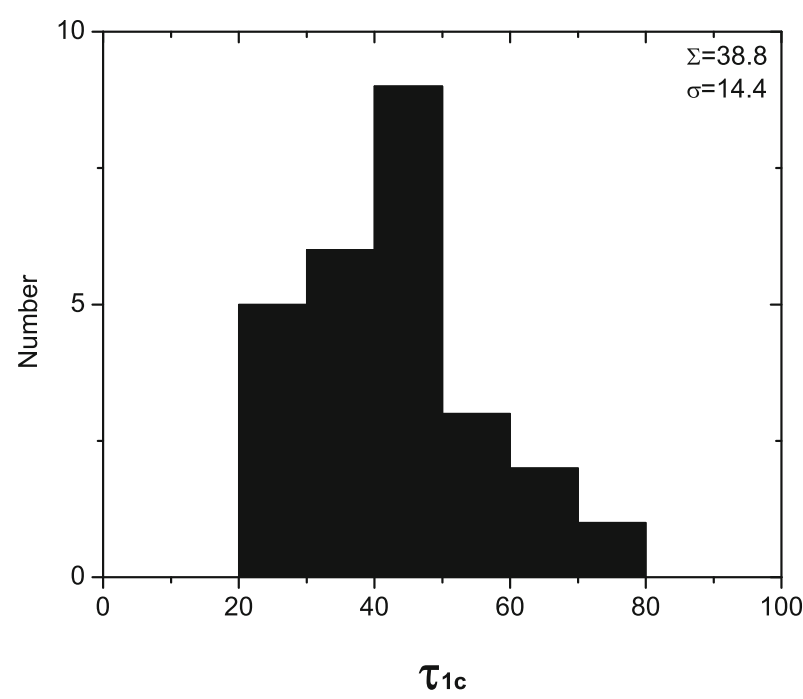

Fig. 4. Distribution of recovery or reconfiguration time $\left(\tau_{3 c}\right)$ determined from convection. Mean and standard deviation of the distribution are shown in the figure.

show much variation after the dipolarization and remains steady at its pre-substorm value. The distribution of the recovery time $\left(\tau_{3 \mathrm{c}}\right)$ determined from convection is shown in Fig. 4. The distribution shows a peak around $47.5 \mathrm{~min}$ and an average of $38.8 \mathrm{~min}$. This is an important time scale since during this time there are no substorms, and this period represents the magnetospheric reconfiguration time during which the magnetosphere attains its pre-substorm state. This distribution is also similar to the recovery time distribution shown by Chua et al. (2004) form the POLAR UVI measurements.

We have shown that the average growth, expansion, and recovery time for the substorm determined from the polar cap convection are 31.6, 22.4, and $38.8 \mathrm{~min}$, respectively, for the 26 substorms studied in this paper. Average growth and expansion time scales determined from the geosynchronous magnetic field measurements are 51.2 and $5.8 \mathrm{~min}$, respectively. The expansion and recovery time scales shown in this study are comparable to those reported by Weimer (1994) and Chua et al. (2004) using different ionospheric measurements. Even though the time scales presented in this paper are important by themselves, the interesting aspect of this study is the comparison of the substorm growth and expansion time scales determined from the polar cap convection with those determined using the geosynchronous magnetic field measurements. The comparison has yielded an interesting result in that the growth time determined using the convection is smaller than the growth time determined using the geosynchronous magnetic field measurements, whereas the expansion time determined using the convection is much greater than the expansion time geosynchronous magnetic field measurements. The examples shown in Figs. 1 and 2 and the distribution shown in Fig. 3 clearly demonstrate this. The difference is between time scales determined using the convection, and the geosynchronous magnetic field measurements for growth and expansion phases are approximately the same (approx. $20 \mathrm{~min}$ ). By looking at the examples shown in 
Figs. 1 and 2, this time difference during the growth phase can be viewed as a delay in setting up the initial ionospheric response to the inner magnetospheric response (ionospheric response was always delayed relative to the inner magnetospheric response in all of the events studied). Once this coupling is established the ionospheric response follows the magnetospheric response until the onset, which is clearly evident in the examples shown in Figs. 1 and 2 (near simultaneous low in the convection and low in the geosynchronous magnetic field inclination). After the onset, the field inclination rises much faster than the convection and this cause the longer expansion time scale that was determined using the convection.

The question is now 'What is the cause of the difference in time scales between the convection and geosynchronous magnetic field measurements?'. There are two probable scenarios: (1) The "fly wheel" effect (Banks, 1972), which is a concept linked to the notion that the neutral winds induced by the ion drag will keep going for a substantial amount of time after the original force is cut off, and (2) inherent time scale in establishing the mechanism that causes the variations in the convection associated with the substorm. The fly wheel effect can be easily ruled out as the cause of the difference in the time scales since ionospheric time scales will be much larger, and observed variations are inconsistent with this explanation. Jayachandran et al. (2003) explained the convection changes associated with the substorms using the changes in the Region 1 currents and the current balance in the magnetotail. Polar cap convection decreases during the growth phase due to the reduction in the Region 1 current to balance the increase of cross tail current. The polar cap convection increases after onset and during the expansion phase due to the increase of Region 1 current as a result of the reduction in cross tail current as the tail becomes more dipole-like. If one follows this explanation, the difference in time scales determined using the convection measurements and the geosynchronous magnetic field measurements during the growth phase is the time (approx. $20 \mathrm{~min}$ ) to establish this connection (growth time). This may be the reason for the delayed response of the ionospheric convection. The greater time response of the ionospheric convection when compared to the geosynchronous magnetic field measurements during the expansion phase also may be due to the larger decay time (20 min) of the coupling system. A similar time difference between the ionospheric convection and the geosynchronous magnetic field measurements further reinforces this idea and implies that $20 \mathrm{~min}$ is the time scale to establish the abovementioned mechanism.

\section{Conclusions}

The auroral substorm time scales determined using the polar cap convection measurements revealed that the average time scales associated with growth, expansion, and recovery phases are $33.6,22.4$, and 38.8 min, respectively. A comparison of the growth and expansion time scales determined using the convection measurement and the geosynchronous magnetic field measurements produced some interesting results. The growth time scales determined from the ionospheric convection measurement are smaller than those of the time scales determined from geosynchronous measurements, whereas the expansion time scales determined using the ionospheric convection are much larger than those of the time scales determined using the geosynchronous measurements. Moreover, the difference between time scales determined using the polar cap convection and the geosynchronous magnetic field measurements for the growth and expansion phases are almost the same (approx. $19 \mathrm{~min}$ for growth and approx. 17 mins for expansion). We attribute this time scale to the establishment and decay of the mechanism that causes the convection changes associated with substorms.

Acknowledgments. The research was supported by grants from the Natural Science and Engineering Research Council (NSERC) of Canada. We acknowledge the Coordinated Data Analysis (CDA) web for the access of the ACE solar wind and magnetic field data. Particle injection data was accessed through the LANL web.

\section{References}

Akasofu, S.-I., The development of the auroral substorms, Planet. Space Sci., 12, 273, 1964.

Banks, P. M., Magnetospheric processes and the behavior of the neutral atmosphere, Space Res., 12, 1051, 1972.

Chua, D., G. Parks, M. Brittnacher, G. Germany, and J. Spann, Auroral substorm time scales: IMF and seasonal variations, J. Geophys. Res., 109, A03207, doi:10.1029/2003JA009951, 2004.

Jayachandran, P. T., J. W. MacDougall, E. F. Donovan, J. M. Ruohoniemi, K. Liou, D. R. Moorcroft, and J.-P. St-Maurice, Substorm associated changes in high-latitude ionospheric convection, Geophys. Res. Lett., 30(20), 2064, doi:10.1029/2003GL017497, 2003.

Kawano, H., G. Le, C. T. Russell, G. Rostoker, M. J. Brittnacher, and G. K. Parks, Substorm-time magnetic field perturbations in the polar magnetosphere: Polar pbservations, Earth Planets Space, 54, 963, 2002. MacDougall, J. W. and P. T. Jayachandran, Polar cap convection relationship with solar wind, Rad. Sci., 36(6), 1869, 2001.

Weimer, D. R., Substorm time constants, J. Geophys. Res., 99(6), 11,005, 1994

P. T. Jayachandran (e-mail: jaya@unb.ca) and J. W. MacDougall 